\title{
Hepatic Manifestations of SARS-CoV-2 Infection (COVID-19)
}

\author{
João Marcello de Araujo Neto ${ }^{1 *}$ and Carlos Eduardo Brandão Mello ${ }^{2}$ \\ ${ }^{1}$ Federal University of Rio de Janeiro, National Institute of Câncer, Brazil \\ ${ }^{2}$ Federal University of the State of Rio de Janeiro, Federal University of Rio de Janeiro, Brazil
}

*Corresponding author: João Marcello de Araujo Neto, Federal University of Rio de Janeiro, National Institute of Câncer, Brazil

\begin{tabular}{lll}
\hline ARTICLE INFO & & ABSTRACT \\
\cline { 1 - 1 } $\begin{array}{l}\text { Received: 幽 July 01, } 2020 \\
\text { Published: 幽 July 16, } 2020\end{array}$ & $\begin{array}{l}\text { Citation: João Marcello de Araujo N, Carlos Eduardo Brandão M. Hepatic Manifestations } \\
\text { of SARS-CoV-2 Infection (COVID-19). Biomed J Sci \& Tech Res 28(5)-2020. BJSTR. } \\
\text { MS.ID.004727. }\end{array}$ \\
\hline
\end{tabular}

\section{Short Communication}

Since December 2019, an outbreak of a new Coronavirus (SARS-CoV-2) started in Wuhan (China) and has become pandemic. The growing number of cases reached alarming proportions in China, Europe, United States of America, and Brazil. Most cases of COVID-19 infection are asymptomatic or have mild symptoms and resolve without the need for specific treatment. However, about $15 \%$ can progress to severe pneumonia and have mortality rates of up to $3 \%$. In general, serious presentations results from pulmonary alveolar damage and severe respiratory failure (SARS).

Liver involvement in COVID-19 infection has been the subject of study in some case series that will be summarized below in 5 distinct topics:

a) Hepatic manifestations in patients without previous liver disease.

b) Hepatic manifestations in patients with previous liver disease.

c) Mechanisms of liver injury.

d) Hepatobiliary and gastrointestinal manifestations.

e) Liver care of special populations.
Hepatic Manifestations in Patients without Previous Liver Disease

The clinical manifestations of SARS-CoV-2 infection are predominantly fever (98\%), fatigue (69\%), dry cough (59\%), anorexia (39\%), myalgia (34\%), dyspnea (31\%) , diarrhea and nausea (10\%), according to a Chinese study that analyzed more than 1.099 patients. In this cohort, $81 \%$ of the patients had mild clinical presentations, $13.8 \%$ were severe cases (respiratory frequency > 30 irpm, Sat $02<93 \%$ and pulmonary infiltrate $>50 \%$ ), and $4.7 \%$ were critically ill patients requiring mechanical ventilation and complicated with kidney failure, shock or multiple organ failure. The frequency of elevation of aminotransferases ranged from $20 \%$ to $53 \%$, with this occurrence being more frequent in patients with more severe clinical manifestations. In mild cases, the increase in liver enzymes seems be transient and without clinical significance. In this scenario, it does not require further investigation or specific treatment. In a preliminary analysis of 1.076 Chinese patients on CUIMC cohort, the frequency of AST or ALT elevations was 33\%. But only $10 \%$ presented with aminotransferases greater than 2 times the upper limit of normality (ULN) and $2.4 \%$ was greater than 5 times the ULN. When AST is higher than ALT, it should raise suspicion of AST elevation originated from other sites outside the liver, mainly muscles. 
In a series of cases, an isolated increase in gammaglutamyl transpeptidase (GGT) was reported in up to $54 \%$ of patients. In most cases it was accompanied by normal alkaline phosphatase. Bile duct cells (cholangiocytes) are known to have great expression of angiotensin-converting enzyme 2 that functions as a viral receptor. Although the increase in GGT has been seen in this pandemic, until now, there is no report of worsening of cholestatic diseases by SARS-CoV-2. Marked increase of bilirubin and reduction of albumin are not common, but it may occur, especially in cases of more severe clinical scenario. Changes in coagulation were also described, such as prolongation of prothrombin time, thrombocytopenia, fibrinolysis, disseminated intravascular coagulation (DIC) and episodes of pulmonary thromboembolism.

\section{Hepatic Manifestations in Patients with Previous Liver Disease}

About 300 million Chinese are infected with hepatitis B (HBV) and $\mathrm{C}$ (HCV) viruses or have chronic liver disease. Thus, it is not surprising that many patients with chronic liver disease were infected with SARS-CoV-2 in China. It is admitted that $2 \%$ to $11 \%$ of COVID-19 patients in China had pre-existing chronic liver diseases. American and British studies suggest that chronic liver diseases are risk factors for mortality on COVID-19 infection. However, it remains controversial. In patients with chronic viral hepatitis B or $\mathrm{C}$, antiviral treatment should be maintained. Patients waiting for hepatitis $\mathrm{C}$ treatment can start it during the pandemic, since followup is kept. Telemedicine can be used to monitor the treatment. Diabetes, obesity and other risk factors for COVID-19 severity are more common in fatty liver disease patients. Particular attention should be given to patients with decompensated Child B or C cirrhosis and those with chronic liver diseases in older age. More data is needed to know if they are susceptible to more aggressive COVID-19. Signs of cirrhosis decompensation such as jaundice, hepatic encephalopathy, ascites or digestive bleeding should be evaluated for the possibility of being related to COVID-19.

\section{Potential Mechanisms of Liver Injury}

Molecular biology studies using PCR techniques revealed the presence of the viral genome in the liver tissue and biliary epithelium. Liver damage in COVID-19 infection can be due to a direct viral cytotoxic effect, but a secondary immune damage can be mediated by the inflammatory storm (bystander hepatitis). The production and release of pro-inflammatory cytokines, such as IL-1 and IL-6, may play an important role on this process. Pathology studies of liver fragments revealed the presence of microvesicular steatosis associated with portal and lobular inflammation. In addition, there is also focal and centrolobular necrosis. Morphological aspects are very similar to those described in non-alcoholic fatty liver disease and sepsis. It is also important to highlight the involvement of hepatic vasculature including acute portal and sinusoidal thrombosis. The drugs used in the management of patients with severe COVID-19 can cause liver damage, including macrolide antibiotics, quinolones and clavulanate. Antivirals such as lopinavir, ritonavir, favipiravir, atazanavir, remdesivir can also promote drug induced liver damage. Chloroquine and hydroxychloroquine and IL-6 inhibitors and immunomodulators, such as tocilizumab, siltuximab and sarilumab also have potential liver toxicity. The possibility of drug interaction should be remembered with the use of antivirals such as ritonavir and alteration of the QT interval with the use of chloroquine / hydroxychloroquine. In the context of severe infection, the differential diagnosis of liver damage should include ischemic hepatitis, sepsis and venous congestion of the liver due to right cardiac overload in patients with severe pulmonary disease [1-10].

\section{Hepatobiliary and Gastrointestinal Manifestations}

The presenting manifestations of COVID-19 are gastrointestinal or hepatobiliary in 2 to $10 \%$ of patients. The main digestive symptoms described are nausea, vomiting, diarrhea and abdominal pain, even in the absence of respiratory symptoms. It is possible that patients with this profile may have a worse outcome. The SARS-CoV-2 viral RNA can be detected in intestinal cells and feces, suggesting a possible, but not confirmed, orofecal transmission. Other forms of rare presentation have already been described, such as abdominal pain in the right upper quadrant simulating acalculous cholecystitis and upper abdominal pain suggesting acute pancreatitis. There are reports of acute appendicitis, mesenteric adenitis and forms of false abdominal emergencies in patients with COVID-19. Thus, we should be aware for COVID-19 during pandemic in patients with symptoms outside the respiratory tract.

\section{Liver Care of Special Populations}

It is still unknown whether immunosuppressed patients with autoimmune diseases or after liver transplantation are at increased risk for COVID-19 infections. Studies with immunosuppressed patients in Italy did not show a higher risk for this population. But American data presents conflicting results. International guidelines suggest that patients on immunosuppression and not affected by COVID 19 should continue to use regular medications in the usual doses during the pandemic. There is a suggestion from American Association for the Study of the Liver (AASLD) to reduce the dosage of corticoids on immunosuppressed patients during COVID-19 infection. But current knowledge of benefits of high doses of corticoids specially on inflammatory phase of COVID-19 may challenge this recommendation. It is of fundamental importance that this population remains regularly monitored by medical teams, maintaining recommendations for home isolation and hygiene routines similar to the general population. It is unknown how COVID-19 infection behaves in patients with primary liver cancer. Most medical societies believe that cancer diagnosis and treatment should not be modified during pandemic. Special populations of liver disease should be educated to recognize COVID-19 symptoms and promptly seek for assistance [11-17].

\section{References}


1. Pan L, Mu Mi, Yang Pengcheng, Sun Yu, Wang Runsheng, et al. (2020) Clinical characteristics of COVID-19 patients with digestive symptoms in Hubei, China: A descriptive, cross-sectional, multicenter study, Am J Gastroenterol.

2. Zhang C, Lei Shi, Fu-Sheng Wang (2020) Liver injury in COVID-19: management and challenges. Lancet Gastroenterol Hepatol 2020.

3. Guan WJ, Ni ZY, Hu Y, Wen hua Liang, Chun quan Ou, et al. (2020) Clinical characteristics of 2019 novel coronavirus infection in China. N Engl J Med.

4. Huang C, Wang Y, Li X, Lili Ren, Jianping Zhao, et al. (2020) Clinical features of patients infected with 2019 novel coronavirus in Wuhan, China. Lancet 395: 497-506.

5. Chen N, Zhou M, Dong X, Jieming Qu, Fengyun Gong, et al. (2020) Epidemiological and clinical characteristics of 99 cases of 2019 novel coronavirus pneumonia in Wuhan, China: A descriptive study. Lancet 395: 507-513.

6. Wang D, Hu B, Hu C, Fang fang Zhu, Xing Liu, et al. Clinical characteristics of 138 hospitalised patients with 2019 novel coronavirus-infected pneumonia in Wuhan, China. JAMA.

7. Shi H, Han X, Jiang N, Yukun Cao, Osamah Alwalid, et al. (2020) Radiological findings from 81 patients with COVID-19 pneumonia in Wuhan, China: a descriptive study. Lancet Infect Dis.

8. Xu XW, Wu XX, Jiang XG, Kai Jin Xu, Ling Jun Ying, et al. Clinical findings in a group of patients infected with the 2019 novel coronavirus (SARSCov-2) outside of Wuhan, China: retrospective case series.

9. Yang X, Yu Y, Xu J, Huaqing Shu, Jia'an Xia, et al. (2020) Clinical course

ISSN: $2574-1241$

DOI: $10.26717 /$ BJSTR.2020.28.004727

João Marcello de Araujo Neto. Biomed J Sci \& Tech Res

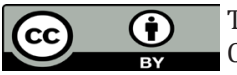

This work is licensed under Creative Commons Attribution 4.0 License

Submission Link: https://biomedres.us/submit-manuscript.php and outcomes of critically ill patients with SARS-CoV-2 pneumonia in Wuhan, China: a single-centered, retrospective, observational study. Lancet Respir Med.

10. Chai X, Hu L, Zhang Y, Weiyu Han, Zhou Lu, et al. (2020) Specific ACE2 expression in cholangiocytes may cause liver damage after 2019-nCoV infection. bioRxiv.

11. Joint GI society message: COVID-19 clinical indights for our community of gastroenterologists and gastroenterology care providers. Disponível em.

12. Gu J, Han B, Wang J (2020) COVID-19: Gastrointestinal manifestations and potential fecal-oral transmission. Gastroenterology. pii: S0016.

13. Xiao F, Tang M, Xiaobin Zheng, Ye Liu, Xiaofeng Li, et al. Evidence for gastrointestinal infection of SARS-CoV-Gastroenterology. 158(6): 18311833.

14. Recomendações da Sociedade Brasileira da SOBED para endoscopia segura durante a pandemia por coronavírus - documento 003/2020 $21 / 02 / 2020$

15. Nota técnica da Sociedade Brasileira de Hepatologia sobre Manifestações Hepáticas na COVID 19. 22 de Março de 2020.

16. D’Antiga (2020) Coronaviruses and imunossupressed patients. Facts during the third epidemic Liver Transplantation 2020.

17. Cardoso F (2020) Liver Transplantation in an ICU dominated by covid-19. Liver transplantation.

$\begin{array}{ll}\text { BIOMEDICAL } & \text { Assets of Publishing with us } \\ \text { RESEARCHES } & \text { - Global archiving of articles } \\ \text { - Immediate, unrestricted online access } & \text { - Rigorous Peer Review Process } \\ & \text { - Authors Retain Copyrights } \\ \end{array}$

